\title{
Differential graded endomorphism algebras, cohomology rings and derived equivalences
}

\author{
SHENGYONG PAN, ZHEN PENG AND JIE ZHANG*
}

\begin{abstract}
In this paper, we will consider derived equivalences for differential graded endomorphism algebras by Keller's approaches. First we construct derived equivalences of differential graded algebras which are endomorphism algebras of the objects from a triangle in the homotopy category of differential graded algebras. We also obtain derived equivalences of differential graded endomorphism algebras from a standard derived equivalence of finite dimensional algebras. Moreover, under some conditions, the cohomology rings of these differential graded endomorphism algebras are also derived equivalent. Then we give an affirmative answer to a problem of Dugas [Dug15] in some special case.
\end{abstract}

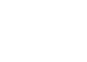

\section{Introduction}

Derived equivalences were introduced by Grothendieck and Verdier and play an important role nowadays in many branches of algebraic geometry, in algebraic analysis, noncommutative algebraic geometry, representation theory, mathematical physics. They capture much of the homological information of an abelian category. For example, the Grothendieck group of an abelian group is preserved under derived equivalences, and derived equivalent algebras have the same Hochschild cohomology.

Important explicit derived equivalences were constructed by Happel [Hap88] in tilting theory and by Rickard [Ric89] in tilting complexes. Keller's ICM talk [Kel06], for instance, justifies derived equivalences by the unbounded derived category of differential graded categories [Kel94]. There are two natural directions of research: How to give systematic methods for constructing derived equivalences, and how to produce new derived equivalences from given ones.

Recently, Hu and Xi constructed [HX13] the derived equivalence for $\Phi$-Auslander-Yoneda algebras. We want to mention that derived equivalence for $\Phi$-Cohen-Macaulay Auslander-Yoneda algebras were also constructed in [Pan14]. Recall that the $\Phi$-Auslander-Yoneda algebras [HX13, Section 3], include, for instance, Auslander algebras, generalized Yoneda algebras and certain trivial extensions. By the definition, $\Phi$-Auslander-Yoneda algebras can be characterized as the cohomology rings of some dg algebras. Hence, it is important to consider derived equivalences for some dg algebras. It is due to Dugas [Dug15, Section 7], who proposed the following problem.

Problem. Let $\Lambda$ and $\Gamma$ be differential graded algebras. If the cohomology rings $H^{*}(\Lambda)$ and $H^{*}(\Gamma)$ are derived equivalent, are the differential graded algebras $\Lambda$ and $\Gamma$ derived equivalent or not.

Motivated by the above problem, we look for in this paper a general way to get derived equivalences of differential graded algebras in two directions.

By considering a triangle

$$
X \longrightarrow Y \longrightarrow Z \longrightarrow X[1]
$$

in the homotopy category $\mathscr{K}(A)$ of a differential graded algebra $A$, we show that there is a derived equivalence between differential graded endomorphism algebras $\mathscr{H} o m(X \oplus Y, X \oplus Y)$ and $\mathscr{H} o m(Y \oplus Z, Y \oplus Z)$. See Theorem 4.7 for more details. Moreover, under some mild conditions, we get the cohomology rings of these differential graded algebra are also derived equivalent. This gives an affirmative answer to the above problem in some special case. Note that it is still an open question whether a derived equivalence of the cohomology rings implies the differential graded algebras are derived equivalent.

\footnotetext{
* Corresponding author. Email: jiezhang @ bit.edu.cn.

2010 Mathematics Subject Classification: primary 18E30, 16G10;16S10,18G15.

Keywords: derived equivalence; differential graded endomorphism algebra; cohomology ring; standard derived equivalence.
} 
We also study a standard derived equivalence $F: \mathscr{D}^{b}(\bmod -B) \longrightarrow \mathscr{D}^{b}(\bmod -C)$ of two finite dimensional algebras $B$ and $C$. By considering the additive functor $\underline{F}: \underline{\bmod }-B \rightarrow \underline{\bmod }-C$ introduced in [HX10, HP17], we get a derived equivalence between differential graded algebras $\mathbf{R} \operatorname{Hom}_{B}(B \oplus X, B \oplus X)$ and $\mathbf{R} \operatorname{Hom}_{C}(C \oplus \underline{F}(X), C \oplus \underline{F}(X))$, where $X$ is a $B$ module (See Theorem 5.3). If $X \in{ }^{\perp} B$, then the cohomology rings $H^{\mathbb{Z}}(A \oplus X)$ and $H^{\mathbb{Z}}(B \oplus \underline{F}(X))$ of $\mathbf{R} \operatorname{Hom}_{A}(A \oplus X, A \oplus X)$ and $\mathbf{R H o m}_{B}(B \oplus \underline{F}(X), B \oplus \underline{F}(X))$, respectively, are derived equivalent by [Pan14, Theorem 1.1]. This gives an affirmative answer to a question of Pan [Pan14, Section 4] which is in spirit to a question of Dugas [Dug15].

This paper is organized as follows. In Section 2 , we recall some basic definitions and facts of derived categories and derived equivalences. Section 3 is devoted to collecting differential graded modules, their derived categories and studying the differential graded bimodules for later use. The construction of derived equivalences from the triangles in the homotopy category of differential graded algebras will be given in Section 4. Finally, in Section 5, we construct derived equivalences for differential graded algebras from standard derived equivalences of finite dimensional algebras.

\section{Preliminaries}

In this section, we recall some basic definitions and collect some basic facts of derived categories and derived equivalences.

In this paper, we fix a commutative ring $k$ with identity. All algebras are $k$-algebras, and functors are $k$-functors. The composite of two morphisms $f: X \rightarrow Y$ and $g: Y \rightarrow Z$ in a category $\mathcal{C}$ will be denoted by $g f$. If $f: X \rightarrow Y$ is a map between two sets, then the image of an element $x \in X$ will be denoted by $f(x)$. The composite of two functors $F: \mathcal{C} \rightarrow \mathcal{D}$ and $G: \mathcal{D} \rightarrow \mathcal{E}$ will be denoted by $G F$. For each object $X$ in $\mathcal{C}$, we write $F(X)$ for the corresponding object in $\mathcal{D}$, and for each morphism $f: X \rightarrow Y$ in $\mathcal{C}$ we write $F(f)$ for the corresponding morphism in $\mathcal{D}$ from $F(X)$ to $F(Y)$.

For an object $M$ in a $k$-category $\mathcal{C}$, we use $\operatorname{add}(M)$ to denote the full subcategory of $\mathcal{C}$ consisting of direct summands of finite direct sums of copies of $M$. If $\mathcal{C}$ admits infinite coproducts, then $\operatorname{Add}(M)$ means the full subcategory of $\mathcal{C}$ consisting of direct summands of all coproducts of copies of $M$.

For generality, we shall consider arbitrary $k$-algebras. All modules will be unitary right modules. Let $A$ be a $k$ algebra. The category of all right $A$-modules will be denoted by Mod- $A$. We use mod- $A$ to denote the full subcategory of Mod- $A$ consisting of finitely presented $A$-modules, that is, $A$-modules $X$ admitting a projective presentation $P_{1} \rightarrow$ $P_{0} \longrightarrow X \rightarrow 0$ with $P_{i}$ finitely generated projective for $i=0,1$. The category of all projective $A$-modules is denoted by Proj- $A$, and the category of all finitely generated projective $A$-modules is denoted by proj- $A$.

Let $A$ be a $k$-algebra, a complex $X^{\bullet}$ over $A$ is a sequences $d_{X}^{i}$ between $A$-modules $X^{i}$ :

$$
\cdots \longrightarrow X^{i-1} \stackrel{d_{X}^{i-1}}{\longrightarrow} X^{i} \stackrel{d_{X}^{i}}{\longrightarrow} X^{i+1} \stackrel{d_{X}^{i+1}}{\longrightarrow} \cdots
$$

such that $d_{X}^{i} d_{X}^{i+1}=0$ for all $i \in \mathbb{Z}$. The category of complexes of $A$-modules, in which morphisms are chain maps, is denoted by $\mathscr{C}(\operatorname{Mod}-A)$, and the corresponding homotopy category is denoted by $\mathscr{K}(\operatorname{Mod}-A)$. We write $\mathscr{D}(\operatorname{Mod}-A)$ for the derived category of Mod- $A$. We also write $\mathscr{K}^{b}(\operatorname{Mod}-A), \mathscr{K}^{-}(\operatorname{Mod}-A)$ and $\mathscr{K}^{+}(\operatorname{Mod}-A)$ for the full subcategories of $\mathscr{K}$ (Mod- $A$ ) consisting of bounded complexes, complexes bounded above, and complexes bounded below, respectively. Denote by $\mathscr{D}^{b}(\operatorname{Mod}-A), \mathscr{D}^{-}(\operatorname{Mod}-A)$ and $\mathscr{D}^{+}(\operatorname{Mod}-A)$ the full subcategories of $\mathscr{D}(\operatorname{Mod}-A)$ consisting of bounded complexes, complexes bounded above, and complexes bounded below, respectively.

Note that there is a fully-faithful functor Mod- $A \longrightarrow \mathscr{D}(\operatorname{Mod}-A)$ by viewing an $A$-module as a complex in $\mathscr{D}($ Mod- $A)$ concentrated in degree zero.

The homotopy category of an additive category, and the derived category of an abelian category are both triangulated categories. For basic facts on triangulated categories, we refer to Neeman's book [Nee01]. The shift functor of a triangulated category will be denoted by [1] in this paper. In the homotopy category, or the derived category of an abelian category, the shift functor acts on a complex by moving the complex to the left by one degree, and changing the sign of the differentials.

Two algebras $A$ and $B$ are said to be derived equivalent if one of the following equivalent conditions holds

(1). $\mathscr{D}($ Mod- $A$ ) and $\mathscr{D}($ Mod- $B)$ are equivalent as triangulated categories.

(2). $\mathscr{D}^{-}($Mod- $A)$ and $\mathscr{D}^{-}($Mod- $B)$ are equivalent as triangulated categories.

(3). $\mathscr{D}^{b}(\operatorname{Mod}-A)$ and $\mathscr{D}^{b}($ Mod- $B)$ are equivalent as triangulated categories.

(4). $\mathscr{K}^{b}$ (Proj- $A$ ) and $\mathscr{K}^{b}$ (Proj- $B$ ) are equivalent as triangulated categories.

(5). $\mathscr{K}^{b}(\operatorname{proj}-A)$ and $\mathscr{K}^{b}$ (proj- $\left.B\right)$ are equivalent as triangulated categories. 
(6). There is a complex $T^{\bullet}$ in $\mathscr{K}^{b}$ (proj- $A$ ) satisfying the conditions:

(a). $\operatorname{Hom}_{\mathscr{K}^{b}(\text { proj-A) }}\left(T^{\bullet}, T^{\bullet}[n]\right)=0$ for all $n \neq 0$,

(b). add $\left(T^{\bullet}\right)$ generates $\mathscr{K}^{b}$ (proj- $A$ ) as a triangulated category,

such that the endomorphism algebra of $T^{\bullet}$ in $\mathscr{K}^{b}(\operatorname{proj}-A)$ is isomorphic to $B$.

For the proof that the above conditions are indeed equivalent, we refer to [Ric89, Kel94]. If the algebras $A$ and $B$ are left coherent rings, then the above equivalent conditions are further equivalent to the following condition.

(7). $\mathscr{D}^{b}(\bmod -A)$ and $\mathscr{D}^{b}(\bmod -B)$ are equivalent as triangulated categories.

Note that in this case the category mod- $A$ of finitely presented $A$-modules is an abelian category. A complex $T^{\bullet}$ satisfying the conditions (6.a) and (6.b) above is called a tilting complex.

A triangle equivalence functor $F: \mathscr{D}(\operatorname{Mod}-A) \rightarrow \mathscr{D}(\operatorname{Mod}-B)$ is called a derived equivalence. It is well-known that the image $F(A)$ is isomorphic in $\mathscr{D}^{b}\left(\operatorname{Mod}-B\right.$ ) to a tilting complex, and there is a tilting complex $T^{\bullet}$ over $A$ such that $F\left(T^{\bullet}\right)$ is isomorphic to $B$ in $\mathscr{D}(\operatorname{Mod}-B)$. The complex $T^{\bullet}$ is called an associated tilting complex of $F$. The following is an easy lemma for the associated tilting complexes. Its proof can be found, for example, in [HX10, Lemma 2.1]

Lemma 2.1. Consider a derived equivalence $F: \mathscr{D}(\operatorname{Mod}-A) \longrightarrow \mathscr{D}($ Mod- $B)$ of two k-algebras $A$ and $B$. Suppose that $F(A)$ is isomorphic in $\mathscr{D}($ Mod- $B)$ to a complex $\bar{T}^{\bullet} \in \mathscr{K}^{b}($ proj- $B)$ of the form

$$
0 \longrightarrow \bar{T}^{0} \longrightarrow \bar{T}^{1} \longrightarrow \cdots \longrightarrow \bar{T}^{n} \longrightarrow 0
$$

for some $n \geq 0$. Then $F^{-1}(B)$ is isomorphic in $\mathscr{D}(\operatorname{Mod}-A)$ to a complex $T^{\bullet} \in \mathscr{K}^{b}(\operatorname{proj}-A)$ of the form

$$
0 \longrightarrow T^{-n} \longrightarrow \cdots \longrightarrow T^{-1} \longrightarrow T^{0} \longrightarrow 0 .
$$

\section{Differential graded algebras and their derived categories}

In this section, we collect some background on differential graded algebras, their derived categories of differential graded modules and differential graded bimodules, which will be used all throughout this paper. Interpreting differential graded algebras as differential graded categories with just one object, the material is a particular case of the development in [Kel94, Kel06, NS14, CY15].

\subsection{Differential graded algebras and differential graded modules}

Recall that a differential graded $k$-algebra (=dg algebra) is a $\mathbb{Z}$-graded associative $k$-algebra $A=\oplus_{i \in \mathbb{Z}} A^{i}$ with a differential $d: A^{i} \rightarrow A^{i+1}$ satisfying the graded Leibniz rule

$$
d(a b)=d(a) b+(-1)^{p} a d(b), \forall a \in A^{p}, b \in A
$$

. A dg right $A$-module is a $\mathbb{Z}$-graded right $A$-module $M=\oplus_{i \in \mathbb{Z}} M^{i}$ with a graded $k$-linear differential $d: M^{i} \rightarrow M^{i+1}$ such that

$$
d(m a)=d(m) a+(-1)^{n} m d(a), \forall m \in M^{n}, a \in A .
$$

It is useful to look at each $\operatorname{dg} A$-module as a complex

$$
\cdots \rightarrow M^{i-1} \stackrel{d^{i-1}}{\rightarrow} M^{i} \stackrel{d^{i}}{\rightarrow} M^{i+1} \rightarrow \cdots
$$

of $k$-modules with some extra properties.

A morphism between right $\operatorname{dg} A$-modules $f: M \rightarrow N$ is a morphism of the underlying graded $A$-modules which is homogeneous of degree zero and commutes with the differential. Denote by Gr- $A$ the category of graded right $A$-modules with graded $A$-module homomorphisms. Let $M$ and $N$ be right $\operatorname{dg} A$-modules. Set $\mathscr{H} o m_{A}(M, N)^{n}=$ $\operatorname{Hom}_{\mathrm{Gr}-A}(M, N<n>)$ for each $n \in \mathbb{Z}$, it consists of $k$-linear maps $f: M \longrightarrow N$ which are homogeneous of degree $n$ and satisfy $f(m a)=f(m) a$ for all homogeneous elements $a \in A$. The graded vector space

$$
\mathscr{H o m}_{A}(M, N)=\oplus_{i \in \mathbb{Z}} \mathscr{H} m_{A}(M, N)^{i}=\oplus_{i \in \mathbb{Z}} \operatorname{Hom}_{\mathrm{Gr}-A}(M, N<i>)
$$

has a natural differential $d$ such that $d(f)=d_{N} \circ f+(-1)^{|f|+1} f \circ d_{M}$. Furthermore, $\mathscr{H}_{0} m_{A}(M, M)$ becomes a dg algebra with this differential and the usual composition as multiplication. Denote by $\mathcal{C}_{d g} A$ the category of differential graded right $A$-modules with morphism space $\mathcal{C}_{d g} A(M, N)=\mathscr{H}_{0} m_{A}(M, N)$. 


\subsection{Derived category of dg module categories}

Let $A$ be a dg $k$-algebra. Let $\mathscr{C}(A)$ be the category of $\operatorname{dg} A$-modules. A morphism $f: X \longrightarrow Y$ in $\mathscr{C}(A)$ is a morphism in $\mathrm{Gr}-A$ which is a chain map of complexes of k-modules. We have

$$
\operatorname{Hom}_{\mathscr{C}(A)}(X, Y)=\mathcal{Z}^{0}\left(\mathscr{H}_{0} m_{A}(X, Y)\right)=\left\{f \in \mathscr{H}_{0} m_{A}(M, N)^{0} \mid d(f)=0\right\} .
$$

Note that $\mathscr{C}(A)$ is an abelian category and comes with a canonical shifting [1] : $\mathscr{C}(A) \longrightarrow \mathscr{C}(A)$ which comes from the canonical shifting of Gr-A, by defining $d_{M[1]}^{n}=d_{M}^{n+1}$ for each $n \in \mathbb{Z}$. Then we have an obvious faithful forgetful functor

$$
F: \mathscr{C}(A) \longrightarrow \mathrm{Gr}-A
$$

which is also dense on objects since we can interpret each graded $A$-module as an object of $\mathscr{C}(A)$ with zero differential. Viewing the objects of $\mathscr{C}(A)$ as complexes of $k$-modules, we clearly have, for each $p \in \mathbb{Z}$, the $p$-th homology functor

$$
H^{p}: \mathscr{C}(A) \longrightarrow \text { Mod- } k
$$

A morphism $f: X \longrightarrow Y$ in $\mathscr{C}(A)$ is called a quasi-isomorphism if $H^{p}(f)$ is an isomorphism, for all $p \in \mathbb{Z}$. A dg A-module $X$ is called acyclic if $H^{p}(X)=0$, for all $p \in \mathbb{Z}$.

For any $\operatorname{dg} A$-module $X=\oplus X^{i}$, we define a functor $F_{\rho}: \operatorname{Gr}-A \longrightarrow \mathscr{C}(A)$ given by

$$
F_{\rho}(X)=X \oplus X<1>
$$

as graded $A$-modules. For a map $f \in \operatorname{Hom}_{\mathrm{Gr}-A}(X, Y), F_{\rho}(f): F_{\rho}(X) \longrightarrow F_{\rho}(Y)$ given by

$$
F_{\rho}\left(\left[\begin{array}{l}
x \\
y
\end{array}\right]\right)=\left[\begin{array}{l}
f(x) \\
f(y)
\end{array}\right] .
$$

Then we get a $\operatorname{dg} A$-module $F_{\rho}(X)=X \oplus X<1>$ given by

$$
\left[\begin{array}{l}
x \\
y
\end{array}\right] a=\left[\begin{array}{c}
x a \\
y a+(-1)^{x \mid x} x d(a)
\end{array}\right]
$$

with the differential $d=\left[\begin{array}{ll}0 & 1 \\ 0 & 0\end{array}\right]$. It is well-known that $\left(F, F_{\rho}\right)$ is an adjoint pair. Note that $F_{\rho} F(X)$ is a projective-injective object in $\mathscr{C}(A)$, and we have a canonical exact sequence $0 \longrightarrow X \longrightarrow F_{\rho} F(X) \longrightarrow X[1] \longrightarrow 0$ which splits in Gr- $A$ but not in $\mathscr{C}(A)$. The exact structure on $\mathscr{C}(A)$ is the exact sequence

$$
0 \longrightarrow X \longrightarrow Y \longrightarrow Z \longrightarrow 0
$$

in $\mathscr{C}(A)$ which is split in Gr- $A$. With this exact structure, $\mathscr{C}(A)$ is a Frobenius exact category. Then the stable category $\mathscr{C}(A)$ with respect to the given exact structure is denoted by $\mathscr{K}(A)$, and is called the homotopy category of $A$. It is a triangulated category, and each triangle in $\mathscr{K}(A)$ is isomorphic to

$$
X \stackrel{f}{\longrightarrow} Y \stackrel{\left[\begin{array}{c}
-1 \\
0
\end{array}\right]}{\longrightarrow} X[1] \oplus Y \stackrel{\left[\begin{array}{ll}
0 & 1
\end{array}\right]}{\longrightarrow} X[1]
$$

for some $f: X \longrightarrow Y$. Let $f: M \rightarrow N$ be a morphism of $\operatorname{dg} A$-modules. We say that $f$ is a null-homotopic if we get

$$
f=d r+r d,
$$

where $r: M \rightarrow N$ is a morphism of the underlying graded $A$-modules which is homogeneous of degree -1 . Denote by $\Sigma$ the class of all homotopy class of quasi-isomorphisms. Then $\Sigma$ is a multiplicative system in $\mathscr{K}(A)$ compatible with the triangulation and the localization

$$
\mathscr{D}(A):=\mathscr{K}(A)\left[\Sigma^{-1}\right]
$$

is called the derived category of $A$. It is also a triangulated category with translation functor [1] induced from $\mathscr{C}(A)$.

For two dg $A$-modules $X$ and $Y$, there is an isomorphism

$$
\operatorname{Hom}_{\mathscr{K}(A)}(X, Y[n]) \simeq H^{n}\left(\mathscr{H}_{0} m_{A}(X, Y)\right)
$$

for each $n \in \mathbb{Z}$. We observe an isomorphism $\mathscr{H}_{0} m_{A}(A, X) \simeq X$ of complexes sending $f$ to $f(1)$. Then the above isomorphism induces the following isomorphism

$$
\operatorname{Hom}_{\mathscr{K}(A)}(A, X[n]) \simeq H^{n}(X) .
$$


It is well-known that $\mathscr{D}(A)$ can be realized as the Verdier quotient category of $\mathscr{K}(A)$ by its full subcategory of acyclic $\operatorname{dg} A$-modules. A dg $A$-module $P$ is called homotopically projective if $\mathscr{H}_{0} m_{A}(P, M)=0$, for every acyclic dg $A$-module $M$, which is equivalent to the condition that the canonical functor $\mathscr{K}(A) \longrightarrow \mathscr{D}(A)$ induces an isomorphism

$$
\operatorname{Hom}_{\mathscr{K}(A)}(P, X) \simeq \operatorname{Hom}_{\mathscr{D}(A)}(P, X)
$$

for any $\operatorname{dg} A$-module $X$.

For a triangulated category $\mathcal{T}$ and a class $\mathcal{S}$ of objects, we denote by thick $\mathcal{T} \mathcal{S}$ the smallest thick subcategory of $\mathcal{T}$ containing $\mathcal{S}$. Denote by $\operatorname{Tria}_{\mathcal{T}} \mathcal{S}$ is the smallest subcategory of $\mathcal{T}$ containing $\mathcal{S}$ and closed under coproducts. Recall that a thick subcategory is a triangulated subcategory which is closed under direct summands. We say an object $M$ in $\mathcal{T}$ is compact if the functor $\operatorname{Hom}_{\mathcal{T}}(M,-)$ commutes with arbitrary coproducts.

The following easy lemma is useful in the later, for the proof we refer to [NS12, Corollary 3.19].

Lemma 3.1. Let $F: \mathcal{T} \longrightarrow \mathcal{T}^{\prime}$ be a triangle functor of triangulated categories and $T \in \mathcal{T}$ an object. Then

$$
F\left(\operatorname{thick}_{\mathcal{T}}(T)\right) \subseteq \operatorname{thick}_{\mathcal{T}},(F(T))
$$

The following is a very well-known fact [Kel94, theorem 5.3].

Proposition 3.2. Let $A$ be a dg algebra. The compact objects of $\mathscr{D}(A)$ are precisely the objects of thick $\mathscr{D}(A)(A):=\operatorname{per} A$.

The triangulated subcategory per $A$ is called the perfect derived category of $A$.

\subsection{Differential graded bimodules}

We give in this subsection some useful lemmas for later use.

Let $A$ and $B$ be two dg algebras. A dg $A$ - $B$-bimodule $X$ is a left dg $A$-module as well as right dg $B$-module such that

$$
d(a m b)=(d a) m b+(-1)^{p} a(d m) b+(-1)^{p+q} a m(d b),
$$

for all $a \in A^{p}, m \in M$ and $b \in B^{q}$. And the canonical map $A \longrightarrow \mathscr{H}_{0} m_{B}(X, X)$, sending $a$ to $l_{a}$ with $l_{a}(x)=a x$ is a homomorphism of dg algebras. Similarly, the canonical map $B \longrightarrow \mathscr{H}_{0} m_{A^{o p}}(X, X)^{o p}$, sending $b$ to $r_{b}$ with $r_{b}(x)=$ $(-1)^{|b||x|} x b$ is a homomorphism of dg algebras.

Recall that a $\operatorname{dg} A$ - $B$-bimodule $X$ is called left quasi-balanced if the canonical map $A \longrightarrow \mathscr{H}_{0} m_{B}(X, X)$ of dg algebras is a quasi-isomorphism. Dually, $X$ is called right quasi-balanced if the canonical map $B \longrightarrow \mathscr{H}_{0} m_{A^{o p}}(X, X)$ of $\mathrm{dg}$ algebras is a quasi-isomorphism. If both the canonical map $A \longrightarrow \mathscr{H}_{0} m_{B}(X, X)$ and the canonical map $B \longrightarrow$ $\mathscr{H}_{0} m_{A^{o p}}(X, X)$ of dg algebras are quasi-isomorphisms, then $X$ is called a quasi-balanced bimodule. Let $X$ be a dg $A-B$ bimodule. Then $\mathscr{H}_{0} m_{B}\left({ }_{A} X_{B}, Y_{B}\right)$ becomes a right dg $A$-module by $(f a)(x)=f(a x)$ for $f \in \mathscr{H}_{0} m_{B}\left({ }_{A} X_{B}, Y_{B}\right)$ and $x \in X$. Similarly, $\mathscr{H} o m\left({ }_{A} X_{B},{ }_{A} Y\right)$ is a left dg $B$-module by $(b f)(x)=(-)^{|x|(|f|+|b|)} f(x b)$ for $f \in \mathscr{H} m_{B}\left({ }_{A} X_{B},{ }_{A} Y\right)$ and $x \in X$.

Here we give a condition for a fully faithful exact functor between triangulated categories to be an equivalence. We also refer the reader to [Bri99, Theorem 3.3].

Lemma 3.3. Let $\mathcal{T}$ and $\mathcal{S}$ be triangulated categories. Let $F: \mathcal{T} \longrightarrow \mathcal{S}$ and $G: \mathcal{S} \longrightarrow \mathcal{T}$ be an adjoint pair of triangle functors. Then we have the following

(1) If the unit $\eta: I d_{\mathcal{T}} \longrightarrow G F$ is an isomorphism and $G X \simeq 0 \Leftrightarrow X \simeq 0$, then $F$ and $G$ induce mutually inverse equivalences between $\mathcal{T}$ and $\mathcal{S}$.

(2) If the counit $\varepsilon: F G \longrightarrow I d_{\mathcal{S}}$ is an isomorphism and $F Y \simeq 0 \Leftrightarrow Y \simeq 0$, then $F$ and $G$ induce mutually inverse equivalences between $\mathcal{T}$ and $\mathcal{S}$.

Proof. (1) For any $Y \in \mathcal{S}$, there is a triangle

$$
F G Y \stackrel{\varepsilon_{Y}}{\longrightarrow} Y \longrightarrow Z \longrightarrow(F G Y)[1]
$$

Applying the functor $G$, then we get the following triangle

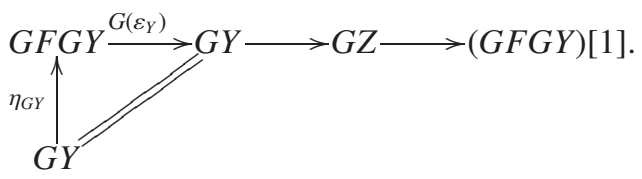


Therefore, we get $1_{G X}=G\left(\varepsilon_{Y}\right) \eta_{G Y}$. Since $\eta_{G Y}: G Y \longrightarrow G F G Y$ is an isomorphism, $G F G Y \stackrel{G\left(\varepsilon_{Y}\right)}{\longrightarrow} G Y$ is an isomorphism. Then $G Z \simeq 0$. By assumptation, $Z \simeq 0$. Consequently, $F G Y \simeq Y$.

(2) The proof of (2) is similar to that of (1).

The following lemma is the Morita theory for derived categories of dg sense which was proved by Keller [Kel94]. We give an another characterization by Lemma 3.3

Lemma 3.4. Let ${ }_{A} M_{B}$ be a $d g A$-B-bimodule such that $M_{B}$ is a homotopically projective compact generator in $\mathscr{D}(B)$ and the canonical map

$$
A \longrightarrow \mathscr{H}_{B}(M, M), \quad(a \mapsto(m \mapsto a m))
$$

is a quasi-isomorphism of dg algebras. Then $-\stackrel{\mathbf{L}}{\otimes}_{A} M$ and $\mathbf{R} \mathscr{H}_{B} m_{B}(M,-)$ induces mutually inverse equivalences between $\mathscr{D}(A)$ and $\mathscr{D}(B)$.

Proof. Let $F=-\otimes_{A} M$ and $G=\mathscr{H}_{0} m_{B}(M,-)$. Then we consider the left and right derived functor $\bar{F}:=-\stackrel{\mathbf{L}}{\otimes_{A}} M$ and $\bar{G}:=\mathbf{R} \mathscr{H}_{0} m_{B}(M,-)$, respectively. We know that $G$ preserves a quasi-isomorphism. Then we have the following

$$
A_{A} \stackrel{\eta_{A}}{\longrightarrow} G F A=\mathscr{H}_{0} m_{B}\left({ }_{A} M_{B}, A \otimes_{A} M\right) \longrightarrow \mathscr{H}_{B} m_{B}(M, M), \quad(a \mapsto(m \mapsto a \otimes m \mapsto a m))
$$

Hence $\eta_{A}$ is a quasi-isomorphism. Since $M_{B}$ is a homotopically projective, the unit of $A \longrightarrow \bar{G} \bar{F} A$ is an isomorphism. Let

$$
\mathscr{X}=\left\{X \in \mathscr{D}(A) \mid \eta_{X}: X \longrightarrow \bar{G} \bar{F} X \text { is an isomorphism }\right\} .
$$

Then $\mathscr{X}$ is a subcategory of $\mathscr{D}(A)$ containing $A_{A}$. Let $\mathscr{K}(A)_{a c}=\{M \in \mathscr{K}(A) \mid M$ is acyclic as $k$-complex $\}$ and $\mathscr{K}(A)_{a c}^{\perp}=\left\{X \in \mathscr{K}(A) \mid \operatorname{Hom}_{\mathscr{K}(A)}\left(\mathscr{K}(A)_{a c}, X\right)=0\right\}$. Then we have the following triangle functors

$$
\operatorname{Tria}(A) \stackrel{\lambda}{\hookrightarrow} \mathscr{K}(A) \stackrel{q}{\longrightarrow} \mathscr{D}(A), \mathscr{K}(A)_{a c}^{\perp} \stackrel{\mu}{\hookrightarrow} \mathscr{K}(A) \stackrel{q}{\longrightarrow} \mathscr{D}(A) .
$$

Here $q \lambda$ and $q \mu$ are equivalent of triangle functors. Let $\gamma$ and $\gamma^{\prime}$ be the quasi-inverse of $q \lambda$ and $q \mu$, respectively. Set $p:=\lambda \gamma$ and $i:=\mu \gamma^{\prime}$. We thus have the following diagram

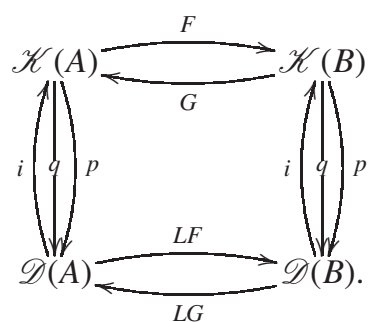

Note that $\bar{F}=q F p$ and $\bar{F}=q G i$. The functors $p, F, q, G$ preserve coproducts. It follows that $i(\coprod X) \simeq i(\coprod q i(X)) \simeq$ $i(q \coprod i(X)) \simeq i q(\coprod i(X)) \simeq \coprod i(X)$. Therefore, $\mathscr{X}$ is closed under coproducts. Consequently, we get Tria $\mathscr{D}(A)(A) \subseteq$ $\mathscr{X} \subseteq \mathscr{D}(A)$. Then $\mathscr{X}=\mathscr{D}(A)$. This implies that $\left.\eta_{X}: X \longrightarrow \bar{G} \bar{F}(X)\right)$ is an isomorphism. For any $X \in \mathscr{D}(B)$ such that $\bar{G}(X)=0$. Thus $q G i(X)=0$. Therefore, $q \mathscr{H} \operatorname{lom}_{B}\left(M_{B}, i(X)\right)=0$. Thus $\mathscr{H}_{0} m_{B}\left(M_{B}, i(X)\right)$ is acyclic. It follows that $\operatorname{Hom}_{\mathscr{K}(B)}\left(M_{B}, i(X)[n]\right)=0$ for $n \in \mathbb{Z}$. So, $\operatorname{Hom}_{\mathscr{D}(B)}\left(M_{B}, X[n]\right)=0$. Then $X \simeq 0$ by $M_{B}$ is a compact generator in $\mathscr{D}(B)$. This completes the proof by Lemma 3.3 .

Corollary 3.5. [Kel94] Suppose that $\phi: A \longrightarrow B$ is a dg algebra homomorphism. If $\phi$ is a quasi-isomorphism of dg algebras, then $-\stackrel{\mathbf{L}}{\otimes}_{A} B$ and $\mathbf{R} \mathscr{H} m_{B}(B,-)$ induces mutually inverse equivalences between $\mathscr{D}(A)$ and $\mathscr{D}(B)$.

Proof. This is the special case of Lemma 3.4 for $M={ }_{A} B_{B},{ }_{A} B_{B}$ is a dg $A$ - $B$-bimodule such that $B_{B}$ is a homotopically projective compact generator in $\mathscr{D}(B)$. 


\section{Derived equivalences of $\mathbf{d g}$-algebras induced from triangles}

Throughout this section, we fix $A$ a dg algebra and denote its homotopy category by $\mathscr{K}(A)$. The goal of this section is to get derived equivalences of two dg algebras which are obtained from some triangles in $\mathscr{K}(A)$. As an application, we give an affirmative answer to Dugas' question [Dug15]. First we need the following well-known observation.

Lemma 4.1. [IT13] Lemma 8.4] Let $F: \mathcal{T} \longrightarrow \mathcal{T}^{\prime}$ be a triangle functor of triangulated categories and $T$ an object in $\mathcal{T}$. If

$$
F_{T, T}: \operatorname{Hom}_{\mathcal{T}}(T, T[n]) \longrightarrow \operatorname{Hom}_{\mathcal{T}^{\prime}}(F(T), F(T)[n])
$$

is an isomorphism for any $n \in \mathbb{Z}$, then

$$
F: \operatorname{thick}_{\mathcal{T}}(T) \longrightarrow \mathcal{T}^{\prime}
$$

is fully faithful.

We generalizes [IT13, Lemma 8.5] to dg algebra case in the following.

Lemma 4.2. Let $X$ be a $d g A$-module and $\Lambda=\mathscr{H}_{0} m_{A}(X, X)$. The we have a triangle equivalence between thick $\mathscr{K}(A)(X)$ and $\operatorname{per} \Lambda$.

Proof. Consider the following functor

$$
\mathscr{H}_{A}(X,-): \mathscr{C}(A) \longrightarrow \mathscr{C}(\Lambda)
$$

which sends $Y$ to a dg $\Lambda$-module $\mathscr{H} o m_{A}(X, Y)$. A map $f: Y_{1} \longrightarrow Y_{2}$ in $\mathscr{C}(A)$ yields

$$
\mathscr{H o m}_{A}(X, f): \mathscr{H o m}_{A}\left(X, Y_{1}\right) \longrightarrow \mathscr{H}_{A}\left(X, Y_{2}\right)
$$

Then this functor sends a null-homotopic morphism of $\mathrm{dg} A$-modules to a null-homotopic morphism of dg $\Lambda$-modules. Therefore, $\mathscr{H}_{0} m_{A}(X,-)$ induces a triangle functor $\mathscr{K}(A) \longrightarrow \mathscr{K}(\Lambda)$. By composing with the canonical functor $\mathscr{K}(\Lambda) \longrightarrow \mathscr{D}(\Lambda)$, we have a triangle functor $\mathscr{K}(A) \longrightarrow \mathscr{D}(\Lambda)$ which sends $X$ to $\Lambda$. We thus get a triangle functor from thick $\mathscr{K}_{(A)}(X)$ to thick $\mathscr{D}(\Lambda)(\Lambda)=\operatorname{per} \Lambda$ by Lemma 4.1. Then we have the following commutative diagram

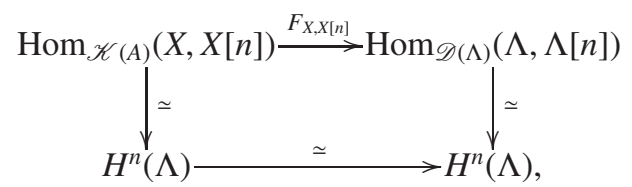

for any $n \in \mathbb{Z}$. It completes the proof by Lemma 4.1

Lemma 4.3. Suppose $X$ and $Y$ are $d g A$-modules such that $Y \in \operatorname{thick}_{\mathscr{K}(A)}(X)$. Let $\Lambda=\mathscr{H}_{0} m_{A}(X, X)$. Then we have the following quasi-isomorphism:

$$
\mathscr{H o m}_{A}(Y, Z) \longrightarrow \mathscr{H}_{\Lambda}\left(\mathscr{H}_{0} m_{A}(X, Y), \mathscr{H o m}_{A}(X, Z)\right) \quad f \mapsto(g \mapsto f \circ g)
$$

for any $d g$ A-module $Z$.

Proof. As in the proof in Lemma 4.2 the functor $\mathscr{H} \operatorname{lom}_{A}(X,-)$ induces a triangle functor $\mathscr{K}(A) \longrightarrow \mathscr{K}(\Lambda)$. Applying the functor $H^{i}$ to

$$
\mathscr{H o m}_{A}(Y, Z) \longrightarrow \mathscr{H}_{\mathrm{O}}\left(\mathscr{H}_{\Lambda} m_{A}(X, Y), \mathscr{H o m}_{A}(X, Z)\right)
$$

it yields that

$$
\operatorname{Hom}_{\mathscr{K}(A)}(Y, Z[i]) \longrightarrow \operatorname{Hom}_{\mathscr{K}(\Lambda)}\left(\mathscr{H} m_{A}(X, Y), \mathscr{H} m_{A}(X, Z)[i]\right)
$$

We now let

$$
\begin{gathered}
\mathscr{X}=\left\{Y \in \mathscr{K}(A) \mid \text { for any object } Z \in \mathscr{K}(A) \text {, the functor } \mathscr{H}_{A}(X,-)\right. \text { induces the following isomorphism: } \\
\left.\qquad \operatorname{Hom}_{\mathscr{K}(A)}(Y, Z[i]) \longrightarrow \operatorname{Hom}_{\mathscr{K}(\Lambda)}\left(\mathscr{H}_{\mathrm{Com}}(X, Y), \mathscr{H}_{\mathrm{O}}(X, Z)[i]\right)\right\} .
\end{gathered}
$$

Then $\mathscr{X}$ is a subcategory of $\mathscr{K}(A)$. Clearly, $\mathscr{X}$ is closed under shifts and direct summands. Suppose that

$$
Y_{1} \longrightarrow Y_{2} \longrightarrow Y_{3} \longrightarrow Y_{1}[1]
$$


is a triangle in $\mathscr{K}(A)$ with $Y_{1}, Y_{2} \in \mathscr{X}$. Then we have the following commutative diagram

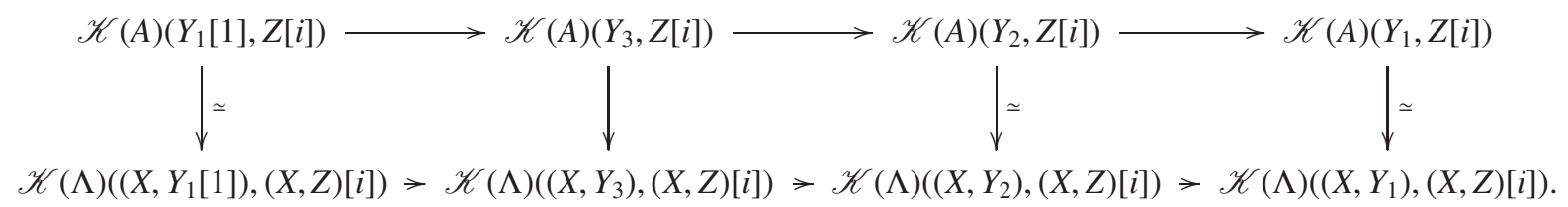

It follows that $Y_{3} \in \mathscr{X}$. Therefore, $\mathscr{X}$ is a thick subcategory of $\mathscr{K}(A)$. If $Y=X$, then

$$
\begin{aligned}
& \operatorname{Hom}_{\mathscr{K}(\Lambda)}\left(\mathscr{H}_{0} m_{A}(X, X), \mathscr{H}_{o m_{A}}(X, Z)[i]\right) \simeq \operatorname{Hom}_{\mathscr{K}(\Lambda)}\left(\Lambda, \mathscr{H}_{o} m_{A}(X, Z)[i]\right) \simeq \operatorname{Hom}_{\mathscr{D}(\Lambda)}\left(\Lambda, \mathscr{H}_{0} m_{A}(X, Z)[i]\right) \\
& \simeq H^{i}\left(\mathscr{H}_{0} m_{A}(X, Z)\right) \simeq \operatorname{Hom}_{\mathscr{K}(A)}(X, Z[i]) \text {. }
\end{aligned}
$$

Thus $X \in \mathscr{X}$. Consequently, thick $\mathscr{K}_{(A)}(X) \subseteq \mathscr{X}$. This completes the proof.

The following lemma is useful, for convenience we give the proof here. We also thank Professor Bernhard Keller for his discussions in the proof of the following lemma.

Lemma 4.4. Suppose that $X$ and $Y$ are $d g$ A-modules such that $Y \in \operatorname{thick}_{\mathscr{K}(A)}(X)$ and $X \in \operatorname{thick}_{\mathscr{K}(A)}(Y)$. Let $\Lambda=$ $\mathscr{H} m_{A}(X, X)$ and $\Gamma=\mathscr{H}_{0} m_{A}(Y, Y)$. Then there is a triangle equivalence between $\mathscr{D}(\Lambda)$ and $\mathscr{D}(\Gamma)$.

Proof. Consider the dg $\Gamma$ - $\Lambda$-bimodule $\mathscr{H} \mathrm{m}_{A}(X, Y)$. By Lemma 4.3 we have the following quasi-isomorphism:

$$
\mathscr{H}_{0} m_{A}(Y, Y) \longrightarrow \mathscr{H}_{0} m_{\Lambda}\left(\mathscr{H} o m_{A}(X, Y), \mathscr{H}_{0} m_{A}(X, Y)\right) \quad f \mapsto(g \mapsto f \circ g)
$$

Similarly to the proof of Lemma 4.3 it follows that

$$
\mathscr{H}_{0} m_{A}(X, X) \longrightarrow \mathscr{H}^{\circ} m_{\Gamma^{o p}}\left(\mathscr{H}_{0} m_{A}(X, Y), \mathscr{H}_{0} m_{A}(X, Y)\right) \quad f \mapsto\left(g \mapsto(-1)^{|f \|| \mid g} g \circ f\right) .
$$

is a quasi-isomorphism of dg algebras. Then the $\mathrm{dg} \Gamma$ - $\Lambda$-bimodule $\mathscr{H}_{0} m_{A}(X, Y)$ is quasi-balanced.

It follows from Lemma 4.2 that $\mathscr{H}_{0} m_{A}(X, Y)_{\Lambda} \in \operatorname{per} \Lambda$ and $\Gamma_{\Gamma} \mathscr{H}_{0} m_{A}(X, Y) \in \operatorname{per} \Gamma^{o p}$, since $Y \in$ thick $_{\mathscr{K}(A)}(X)$ and $X \in \operatorname{thick}_{\mathscr{K}(A)}(Y)$. So $\Lambda \in \operatorname{thick}_{\mathscr{D}(\Lambda)}\left(\mathscr{H}_{0} m_{A}(X, Y)_{\Lambda}\right)$. Then thick $\mathscr{D}(\Lambda)\left(\mathscr{H}_{0} m_{A}(X, Y)_{\Lambda}\right)=\operatorname{per} \Lambda$. Consequently, $\mathscr{H} 0 m_{A}(X, Y)$ is a compact generator for $\mathscr{D}(\Lambda)$. By Lemma 3.4, we have a triangle equivalence between $\mathscr{D}(\Lambda)$ and $\mathscr{D}(\Gamma)$ defined by the dg $\Gamma$ - $\Lambda$-bimodule $\mathscr{H}_{0} m_{A}(X, Y)$.

Corollary 4.5. [ELO09 Proposition 8.3] or [PSY14 Proposition 3.3]. Assume that dg A-modules $X$ and $Y$ are homotopy equivalent. Then the dg algebras $\Lambda=\mathscr{H}_{0} m_{A}(X, X), \Gamma=\mathscr{H}$ om $(Y, Y)$ are quasi-isomorphic. Moreover, we have a triangle equivalence $\mathscr{D}(\Lambda) \simeq \mathscr{D}(\Gamma)$.

Remark 4.6. Let $A$ be a finite dimensional k-algebra, and $X$ be a finitely generated A-module. Let $P_{X}^{\bullet}$ be a projective resolution of $X$. Then we have $\mathbf{R H o m}_{A}(X, X)=\operatorname{Hom}_{A}{ }^{\bullet}\left(P_{X}^{\bullet}, P_{X}^{\bullet}\right)$, where $\mathbf{R H o m}_{A}(X,-)$ is the right derived functor. Suppose $P_{X}{ }^{\bullet}$ and $Q_{X}{ }^{\bullet}$ are two projective resolutions of $X$ which are homotopy equivalent, and let $\Lambda_{1}=\operatorname{Hom}_{A}{ }^{\bullet}\left(P_{X}{ }^{\bullet}, P_{X}{ }^{\bullet}\right)$ and $\Lambda_{2}=\operatorname{Hom}_{A}{ }^{\bullet}\left(Q_{X}{ }^{\bullet}, Q_{X}{ }^{\bullet}\right)$ be two dg algebras. Then we have a triangle equivalence between $\mathscr{D}\left(\Lambda_{1}\right)$ and $\mathscr{D}\left(\Lambda_{2}\right)$ by Lemma4.5

Proof. We give a new proof of $\mathscr{D}(\Lambda) \simeq \mathscr{D}(\Gamma)$ by Lemma 4.4 for reader's convenience. Since $\operatorname{dg} A$-modules $X$ and $Y$ are homotopy equivalent,

$$
\operatorname{thick}_{\mathscr{K}(A)}(X)=\operatorname{thick}_{\mathscr{K}(A)}(Y) .
$$

Let $\Lambda=\mathscr{H}_{0} m_{A}(X, X)$ and $\Gamma=\mathscr{H}_{0} m_{A}(Y, Y)$. There is a dg $\Gamma$ - $\Lambda$-bimodule $\mathscr{H}_{0} m_{A}(X, Y)$ which yields a triangle equivalence between $\mathscr{D}(\Lambda)$ and $\mathscr{D}(\Gamma)$ by Lemma 4.4

The following is the main result in this section.

Theorem 4.7. Let $M$ be a dg A-module in homotopy category $\mathscr{K}(A)$ of $d g$ algebra $A$ and

$$
X \stackrel{f}{\longrightarrow} M_{1} \stackrel{g}{\longrightarrow} Y \stackrel{h}{\longrightarrow} X[1]
$$

be a triangle in $\mathscr{K}(A)$ with $M_{1} \in<M>$, where $<M>$ denotes the full additive subcategory generated by $\cup_{i \in \mathbb{Z}}$ add $M[i]$. Then there is a derived equivalence of dg algebras $\mathscr{H} o m(X \oplus M, X \oplus M)$ and $\mathscr{H} o m(Y \oplus M, Y \oplus M)$. 
Proof. Suppose that $M$ is a $\operatorname{dg} A$-module in $\mathscr{K}(A)$. Let

$$
X \stackrel{f}{\longrightarrow} M_{1} \stackrel{g}{\longrightarrow} Y \stackrel{h}{\longrightarrow} X[1]
$$

be a triangle in $\mathscr{K}(A)$ with $M_{1} \in<M>$. Then there is a triangle

$$
X \stackrel{f}{\longrightarrow} M_{1} \oplus M \stackrel{g}{\longrightarrow} Y \oplus M \stackrel{h}{\longrightarrow} X[1] .
$$

Therefore, $Y \oplus M \in \operatorname{thick}(X \oplus M)$ and $X \oplus M \in \operatorname{thick}(Y \oplus M)$. Set $U=X \oplus M$ and $V=Y \oplus M$. By Lemma4.4, the dg bimodule $\mathscr{H}_{0} m_{A}(U, V)$ defines a derived equivalence between $\mathscr{H} o m(X \oplus M, X \oplus M)$ and $\mathscr{H} o m(Y \oplus M, Y \oplus M)$.

By Theorem 4.7] and [Dug15, Theorem 4.1], we can restate the above theorem for the case of a $k$-algebra $A$.

Corollary 4.8. Suppose that $A$ is a k-algebra and $M^{\bullet}$ is a complex in $\mathscr{K}(A)$. Let

$$
X^{\bullet} \stackrel{f^{\bullet}}{\longrightarrow} M_{1} \stackrel{g^{\bullet}}{\rightarrow} Y^{\bullet} \stackrel{h^{\bullet}}{\longrightarrow} X^{\bullet}[1]
$$

be a triangle in $\mathscr{K}(A)$ with $M_{1}^{\bullet} \in<M^{\bullet}>$. Assume that $f^{\bullet}$ is a left $<M^{\bullet}>$-approximation of $X^{\bullet}$ and $g^{\bullet}$ is a right $<M^{\bullet}>$-approximation of $Y^{\bullet}$. Then

(1). There is a derived equivalence between dg algebras $\operatorname{Hom}_{A}{ }^{\bullet}\left(X^{\bullet} \oplus M^{\bullet}, X^{\bullet} \oplus M^{\bullet}\right)$ and $\operatorname{Hom}_{A}{ }^{\bullet}\left(Y^{\bullet} \oplus M^{\bullet}, Y^{\bullet} \oplus M^{\bullet}\right)$.

(2). The cohomology rings $H^{\mathbb{Z}}\left(X^{\bullet} \oplus M^{\bullet}\right)$ and $H^{\mathbb{Z}}\left(Y^{\bullet} \oplus M^{\bullet}\right)$ are also derived equivalent, where $H^{\mathbb{Z}}\left(X^{\bullet} \oplus M^{\bullet}\right)=$ $\bigoplus_{i \in \mathbb{Z}} \operatorname{Hom}_{\mathscr{K}(A)}\left(X^{\bullet} \oplus M^{\bullet},\left(X^{\bullet} \oplus M^{\bullet}\right)[i]\right)$.

Remark 4.9. There is a derived equivalence between dg algebras $\operatorname{Hom}_{A}{ }^{\bullet}\left(X^{\bullet} \oplus M^{\bullet}, X^{\bullet} \oplus M^{\bullet}\right)$ and $\operatorname{Hom}_{A}{ }^{\bullet}\left(Y^{\bullet} \oplus M^{\bullet}, Y^{\bullet} \oplus\right.$ $\left.M^{\bullet}\right)$. Under some mild conditions, we get the cohomology rings of these dg algebra are derived equivalent. This gives an affirmative answer to a problem of Dugas [Dug15] in some special case.

Corollary 4.10. Dug15. Theorem 5.2] Let A be a symmetric algebra and $X^{\bullet}$ and $M^{\bullet}$ be any complexes in $\mathscr{K}^{b}(\operatorname{proj}-A)$.

Then there exists a left $<M^{\bullet}>$-approximation $X^{\bullet} \stackrel{f^{\bullet}}{\longrightarrow} M^{\bullet}$ of $X^{\bullet}$ in $\mathscr{K}^{b}(A)$. If $Y^{\bullet}=\operatorname{cone}\left(f^{\bullet}\right)$, we get the derived equivalence between $\operatorname{Hom}_{A}{ }^{\bullet}(X \oplus M, X \oplus M)$ and $\operatorname{Hom}_{A} \bullet(Y \oplus M, Y \oplus M)$.

Proof. Let $\widetilde{\mathscr{K}(A)}$ denote the orbit category $\mathscr{K}(A) /[1]$. Then there is a triangle

$$
X^{\bullet} \stackrel{f^{\bullet}}{\longrightarrow} M^{\prime} \stackrel{g^{\bullet}}{\longrightarrow} Y^{\bullet} \stackrel{h}{\longrightarrow} X^{\bullet}[1]
$$

such that $M^{\prime} \in \operatorname{add}_{\mathscr{\mathscr { K }}(A)} M, f^{\bullet}$ is a left $<M^{\bullet}>$-approximation and $g^{\bullet}$ is a right $<M^{\bullet}>$-approximation by Dug15. Theorem 4.1]. It completes the proof by Theorem 4.7

Example 4.11. [Dug15. Section 7] We now show how to realize dg algebras in one concrete case. Set

$$
A=k[x, y] /\left(x^{n}-y^{s}, x y\right)
$$

and consider

$$
T^{\bullet}=A \stackrel{(x, 0)}{\longrightarrow} A \oplus A
$$

concentrated in degree -1 and 0 . Write $T^{\bullet}=T_{1}^{\bullet} \oplus T_{2}^{\bullet}$, where

$$
T_{1}^{\bullet}:=(0 \stackrel{0}{\longrightarrow} A) \text { and } T_{2}^{\bullet}:=(A \stackrel{x}{\longrightarrow} A) .
$$

Then we conclude that

$$
T_{2}^{\bullet} \stackrel{\left[\begin{array}{l}
\gamma \\
\gamma \epsilon
\end{array}\right]}{\longrightarrow} T_{1}^{\bullet[1]} \oplus T_{1}^{\bullet}
$$

is a left $<T_{1}^{\bullet}>$-approximation, yielding the following triangle in $\mathscr{K}^{b}(\operatorname{proj}-A)$

$$
(A \stackrel{x}{\longrightarrow} A) \stackrel{(1, y)}{\longrightarrow}(A \stackrel{0}{\longrightarrow} A) \stackrel{(x, 1)}{\longrightarrow}(A \stackrel{y}{\longrightarrow} A) \longrightarrow(A \stackrel{x}{\longrightarrow} A)[1],
$$


where

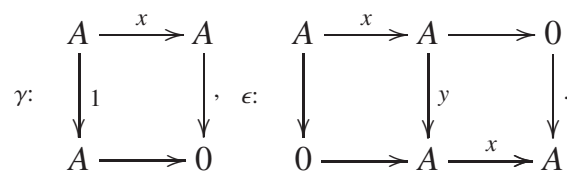

Theorem 4.7 thus shows that two dg algebras

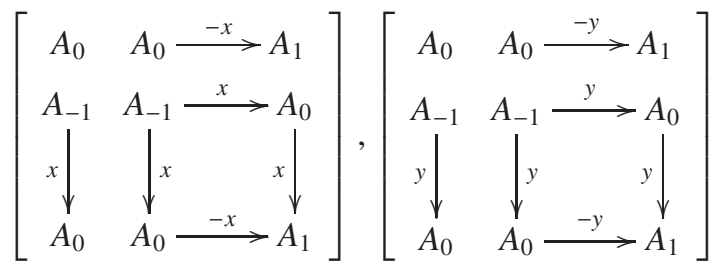

are derived equivalent, where $A_{-1}=A_{0}=A_{1}=A$ concentrate in degree $-1,0,1$, respectively.

\section{Derived equivalence of dg-algebras induced from standard derived equiv- alences of finite dimensional algebras}

Starting from a standard derived equivalence of finite dimensional algebras, we construct in this section derived equivalences of two dg algebras. Moreover, under some conditions, the cohomology rings of these dg algebra are also derived equivalent.

Throughout this section, suppose $k$ is a field. Let $A$ and $B$ be finite dimensional $k$-algebras. Recall that a standard derived equivalence between derived categories $\mathscr{D}^{-}(\operatorname{Mod}-A)$ and $\mathscr{D}^{-}(\operatorname{Mod}-B)$ is an exact functor such that it is an equivalence and is isomorphic to $\operatorname{RHom}\left(X^{\bullet},-\right)$ for some object $X^{\bullet}$ of $\mathscr{D}^{b}(\operatorname{Mod}-A \otimes B)$ [Ric91]. An object $X^{\bullet}$ of $\mathscr{D}^{b}(\operatorname{Mod}-A \otimes B)$ is called a two-sided tilting complex if it induces such an equivalence.

Lemma 5.1. HX10 Lemma 2.1] Let $F: \mathscr{D}(\operatorname{Mod}-A) \longrightarrow \mathscr{D}(\operatorname{Mod}-B)$ be a derived equivalence. For each A-module $X$, the image $F(X)$ is isomorphic, in $\mathscr{D}(\operatorname{Mod}-B)$, to a complex $\bar{T}_{X}^{*}$ of the form

$$
0 \longrightarrow \bar{T}_{X}^{0} \longrightarrow \bar{T}_{X}^{1} \longrightarrow \cdots \longrightarrow \bar{T}_{X}^{n} \longrightarrow 0
$$

with $\bar{T}_{X}^{i}$ projective for all $1 \leq i \leq n$. Moreover, if $X$ admits a projective resolution $P_{X}^{\bullet}$ with $P_{X}^{i}$ finitely generated for $0 \leq i \leq m$ with $m \geq n$. Then $\bar{T}_{X}^{i}$ can be chosen to be finitely generated for all $0 \leq i \leq n$ and $\bar{T}_{X}^{0}$ admits a projective resolution $Q^{\bullet}$ where $Q^{i}$ is finitely generated for $-m \leq i \leq 0$.

Lemma 5.2. $\left[H X 10\right.$ Proposition 3.4] Let $F: \mathscr{D}^{b}(\bmod -A) \longrightarrow \mathscr{D}^{b}(\bmod -B)$ be a derived equivalence. Then there is an additive functor

$$
\underline{F}: \underline{\bmod }-A \rightarrow \underline{\bmod }-B
$$

sending $X$ to $\underline{F}(X)$, such that the following diagram

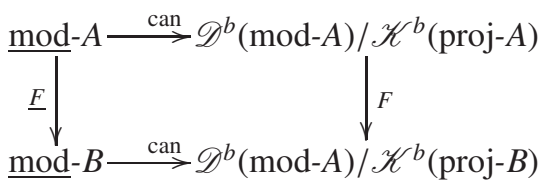

is commutative up to natural isomorphism.

The following theorem is the main result of this section.

Theorem 5.3. Suppose $k$ is a field. Let $F: \mathscr{D}(\operatorname{Mod}-A) \longrightarrow \mathscr{D}(\operatorname{Mod}-B)$ be a standard derived equivalence and

$$
\underline{F}: \underline{\bmod }-A \longrightarrow \underline{\bmod }-B
$$

be the additive functor induced by $F$ as in Lemma 5.2 Then there is a derived equivalence between dg algebras $\mathbf{R H o m}_{A}(A \oplus X, A \oplus X)$ and $\mathbf{R} \operatorname{Hom}_{B}(B \oplus \underline{F}(X), B \oplus \underline{F}(X))$ for each $A$-module $X$. 
Remark 5.4. If $X \in{ }^{\perp} A$, then there is a derived equivalence between dg algebras $\mathbf{R H o m}_{A}(A \oplus X, A \oplus X)$ and $\mathbf{R} \operatorname{Hom}_{B}(B \oplus$ $\underline{F}(X), B \oplus \underline{F}(X))$ by Theorem 5.3 Moreover, the cohomology rings $H^{\mathbb{Z}}(A \oplus X)$ and $H^{\mathbb{Z}}(B \oplus \underline{F}(X))$ of $\mathbf{R} H o \operatorname{H}_{A}(A \oplus X, A \oplus X)$ and $\mathbf{R} \operatorname{Hom}_{B}(B \oplus \underline{F}(X), B \oplus \underline{F}(X))$, respectively, are derived equivalent by [Pan14 Theorem 1.1].

Proof. Let $U=A \oplus X$ and $V=B \oplus \underline{F}(X)$. And let $P_{U}^{\bullet}$ and $P_{V}^{\bullet}$ be projective resolutions of $U$ and $V$, respectively. Then we have $\mathbf{R H o m}_{A}(U, U)=\operatorname{Hom}_{A}{ }^{\bullet}\left(P_{U}^{\bullet}, P_{U}^{\bullet}\right)$ and $\mathbf{R H o m}_{A}(V, V)=\operatorname{Hom}_{A}^{\bullet}\left(P_{V}^{\bullet}, P_{V}^{\bullet}\right)$. By Remark 4.6 the dg endomorphism algebras $\operatorname{Hom}_{A}{ }^{\bullet}\left(P_{U^{\bullet}}, P_{U^{\bullet}}\right)$ and $\operatorname{Hom}_{A}{ }^{\bullet}\left(P_{V} \bullet, P_{V}^{\bullet}\right)$ are unique under derived equivalences. Let $\Lambda=\operatorname{Hom}_{A}{ }^{\bullet}\left(P_{U}{ }^{\bullet}, P_{U} \bullet\right)$ and $\Gamma=\operatorname{Hom}_{A}{ }^{\bullet}\left(P_{V}{ }^{\bullet}, P_{V}{ }^{\bullet}\right)$. It suffices to show that dg algebras $\Lambda$ and $\Gamma$ are derived equivalent.

Let $\bar{T}_{U}^{\bullet}=F(U)$ and $P_{\bar{T}_{U}^{\bullet}}^{\bullet}$ be a projective resolution of $\bar{T}_{U}^{\bullet}$. Set $\Lambda^{\prime}=\operatorname{Hom}_{B}{ }^{\bullet}\left(P_{\bar{T}_{U}^{\bullet}}^{\bullet}, P_{\bar{T}_{U}^{\bullet}}^{\bullet}\right)$. We claim that there is a derived equivalence between $\Lambda^{\prime}$ and $\Gamma$. In the following, we are going to prove the claim.

It follows from Lemma 5.1 that $\bar{T}_{U}^{\bullet}$ is isomorphic, in $\mathscr{D}(B$-mod), to a complex of the form

$$
0 \longrightarrow \bar{T}_{U}^{0} \longrightarrow \bar{T}_{U}^{1} \longrightarrow \cdots \longrightarrow \bar{T}_{U}^{n} \longrightarrow 0
$$

with $\bar{T}_{U}^{i}$ projective for $1 \leq i \leq n, \bar{T}_{U}^{0}=\bar{T}^{0} \oplus \underline{F}(X)$ and $\bar{T}^{0}$ is the first term of $\bar{T}^{\bullet}$, where $\bar{T}^{\bullet}$ is a tilting complex for $B$ by Lemma 2.1, we see that add $\bar{T}^{\bullet}$ generates $\overline{\mathscr{K}}^{b}\left(\operatorname{add}_{B} B\right)$ as triangulated category. All the terms of $\bar{T}^{\bullet}$ are in add $B$. From the distinguished triangle

$$
\bar{T}^{+} \rightarrow \bar{T}_{U}^{\bullet} \rightarrow \bar{T}_{U}^{0} \rightarrow \bar{T}_{U}^{+}[1]
$$

it follows that $\bar{T}_{U}^{0}$ is in the triangulated subcategory generated by add $\left(\bar{T}_{U}^{\bullet}\right)$. Therefore, add $\bar{T}_{U}^{\bullet} \operatorname{generates} \mathscr{K}^{b}\left(\operatorname{add}{ }_{B} V\right)$ as a triangulated category. Consequently, thick $\mathscr{K}_{(B)}\left(\bar{T}_{U}^{\bullet}\right)=\operatorname{thick}_{\mathscr{K}(B)}(V)$. The definition of $P_{\bar{T}_{U}^{\bullet}} \operatorname{implies}_{\text {thick }}(B)\left(P_{\bar{T}_{U}^{\bullet}}\right)=$ thick $\mathscr{D}(B)_{\left(\bar{T}_{U}^{\bullet}\right) \text {. Similarly, thick } \mathscr{D}(B)}\left(P_{V}^{\bullet}\right)=\operatorname{thick}_{\mathscr{D}(B)}(V)$. The canonical functor $\mathscr{K}(B) \stackrel{q}{\longrightarrow} \mathscr{D}(B)$ induces an equivalence between thick $\left.\mathscr{K}(B)_{\left(\bar{T}_{U}\right.}\right)$ and thick ${ }_{\mathscr{D}(B)}^{\bullet}\left(P_{\bar{T}_{U}^{\bullet}}^{\bullet}\right)$ by [HK03, Remark 1.7]. Since $V \in \operatorname{thick}_{\mathscr{K}(B)}(V)=\operatorname{thick}_{\mathscr{K}(B)}\left(\bar{T}_{U}^{\bullet}\right)$, Lemma 3.1 shows $V \in \operatorname{thick}_{\mathscr{D}(B)}\left(\bar{T}_{U}^{\bullet}\right)$. Thus we get $\operatorname{thick}_{\mathscr{D}(B)}(V) \subseteq \operatorname{thick}_{\mathscr{D}(B)}\left(\bar{T}_{U}^{\bullet}\right)=\operatorname{thick}_{\mathscr{D}(B)}\left(P_{\bar{T}_{U}^{\bullet}}^{\bullet}\right)$. Consequently, we obtain thick ${ }_{\mathscr{D}(B)}\left(P_{V}^{\bullet}\right)=\operatorname{thick}_{\mathscr{D}(B)}(V) \subseteq \operatorname{thick}_{\mathscr{D}(B)}\left(P_{\bar{T}_{U}^{\bullet}}^{)}\right)$It follows from [HK03, Remark 1.7] that thick $\mathscr{K}_{(B)}\left(P_{V}^{\bullet}\right) \subseteq$

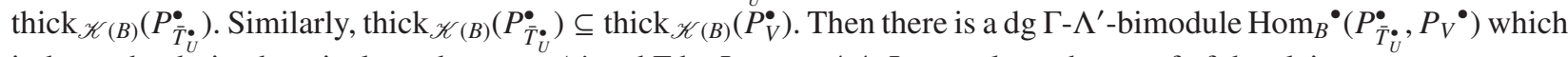
induces the derived equivalence between $\Lambda^{\prime}$ and $\Gamma$ by Lemma 4.4. It completes the proof of the claim.

Finally, it suffices to show that $\Lambda$ and $\Lambda^{\prime}$ are derived equivalent. Since $F$ is a standard derived equivalence, we write $F \simeq Y^{\bullet} \otimes_{A}^{L}-$, where $Y^{\bullet}$ is a bounded above complex of projective $B$ - $A$-bimodules [Ric91]. Then

$$
\mathbf{R H o m}_{B}\left(\bar{T}_{U}^{\bullet}, \bar{T}_{U}^{\bullet}\right) \simeq \operatorname{Hom}_{B}^{\bullet}\left(Y^{\bullet} \otimes_{A}^{L} P_{U}^{\bullet}, Y^{\bullet} \otimes_{A}^{L} P_{U}^{\bullet}\right)=\operatorname{Hom}_{B}^{\bullet}\left(Y^{\bullet} \otimes_{A} P_{U}^{\bullet}, Y^{\bullet} \otimes_{A} P_{U}^{\bullet}\right) \simeq \oplus_{n \in \mathbb{Z}} \sqcap_{i \in \mathbb{Z}} \operatorname{Hom}_{B}\left(M^{p}, M^{p+n}\right) .
$$

We set $M^{\bullet}=Y^{\bullet} \otimes_{A}^{L} P_{U}^{\bullet}$ and then $M^{p}=\oplus_{i \in \mathbb{Z}} Y^{p-i} \otimes_{A} P_{U}^{i}$. Thus, we have

$$
\operatorname{Hom}_{B}\left(M^{p}, M^{p+n}\right)=\operatorname{Hom}_{B}\left(\oplus_{i \in \mathbb{Z}} Y^{p-i} \otimes_{A} P_{U}^{i}, \oplus_{i \in \mathbb{Z}} Y^{p-i} \otimes_{A} P_{U}^{n+i}\right) .
$$

Now, we can define a dg algebra homomorphism $\operatorname{Hom}_{A}{ }^{\bullet}\left(P_{U}^{\bullet}, P_{U}^{\bullet}\right) \longrightarrow \operatorname{Hom}_{B}^{\bullet}\left(M^{\bullet}, M^{\bullet}\right)$ defined by $\left(f^{i}\right) \rightarrow\left(g^{p}\right)$, where $g^{p}=\left(Y^{p-i} \otimes f^{i}\right)$. Indeed, we the above dg algebra homomorphism is a chain map between complexes. Then we get the following commutative diagram

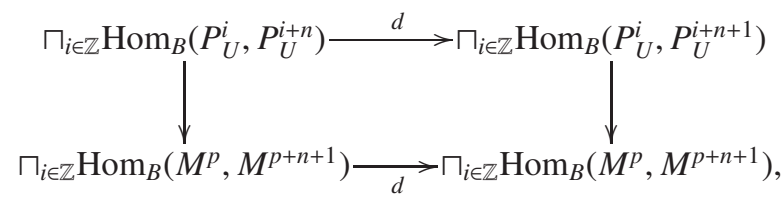

chasing by the following diagram

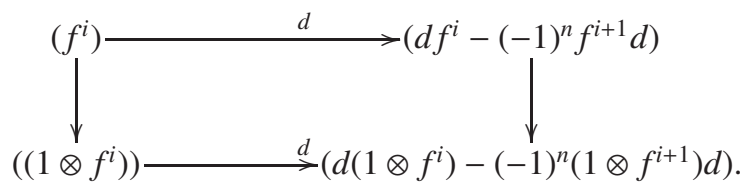

That is, $d f^{i}-(-1)^{n} f^{i+1} d \longrightarrow\left((1 \otimes d)-(-1)^{n}\left(1 \otimes f^{i+1}\right)(1 \otimes d),(d \otimes 1)-(-1)^{n}\left(1 \otimes f^{i}\right)(d \otimes 1)\right)$. Therefore, the dg algebras $\operatorname{Hom}_{A}{ }^{\bullet}\left(P_{U}^{\bullet}, P_{U}^{\bullet}\right)$ and $\operatorname{Hom}_{B}{ }^{\bullet}\left(M^{\bullet}, M^{\bullet}\right)$ are quasi-isomorphic. It follows that $\Lambda$ and $\Lambda^{\prime}$ are quasi-isomorphic. Then the $\operatorname{dg}$ algebras $\Lambda$ and $\Lambda^{\prime}$ are derived equivalent by Corollary 3.5. By the above argument, we conclude that the dg algebras $\Lambda$ and $\Gamma$ are derived equivalent. 
Example 5.5. Let $A=k[x] /\left(x^{n}\right)$. Then $A$ is a representation-finite self-injective algebra. Denote the indecomposable A-module by

$$
X_{r}:=k[x] /\left(x^{r}\right)
$$

for $r=1,2, \cdots, n$. Theorem 5.3 thus shows that two dg algebras $\mathbf{R H o m}_{A}\left(A \oplus X_{r}, A \oplus X_{r}\right)$ and $\mathbf{R} H_{0}{ }_{A}\left(A \oplus X_{n-r}, A \oplus X_{n-r}\right)$ are derived equivalent with $\underline{F}\left(X_{r}\right)=\Omega\left(X_{r}\right)=X_{n-r}$. Take the minimal projective

$$
P_{X_{r}}^{\bullet}=: \cdots \longrightarrow A \stackrel{f_{r}}{\longrightarrow} A \stackrel{f_{n-r}}{\longrightarrow} A \stackrel{f_{r}}{\longrightarrow} A \stackrel{f_{n-r}}{\longrightarrow} A \longrightarrow 0
$$

and

$$
P_{X_{n-r}}^{\bullet}=: \cdots \longrightarrow A \stackrel{f_{n-r}}{\longrightarrow} A \stackrel{f_{r}}{\longrightarrow} A \stackrel{f_{n-r}}{\longrightarrow} A \stackrel{f_{r}}{\longrightarrow} A \longrightarrow 0
$$

of $X_{r}$ and $X_{n-r}$, respectively, where $f_{r}: X_{n} \longrightarrow X_{n}$ by $1+\left(x^{n}\right) \mapsto x^{n-r}+\left(x^{n}\right)$ and $f_{n-r}: X_{n} \longrightarrow X_{n}$ defined by $1+\left(x^{n}\right) \mapsto$ $x^{r}+\left(x^{n}\right)$ see [Zhe15] for more details. Then two dg algebras $\operatorname{Hom}_{A}^{\bullet}\left(A \oplus P_{X_{r}}^{\bullet}, A \oplus P_{X_{r}}^{\bullet}\right)$ and $\operatorname{Hom}_{A}^{\bullet}\left(A \oplus P_{X_{n-r}}^{\bullet}, A \oplus P_{X_{n-r}}^{\bullet}\right)$ are derived equivalent.

It is known that the $\mathbb{N}$-Auslander-Yoneda algebras $\mathrm{E}^{\mathbb{N}}\left(A \oplus X_{r}\right)$ and $\mathrm{E}^{\mathbb{N}}\left(A \oplus X_{n-r}\right)$ are derived equivalent, and they are described in terms of quivers with relations [Zhe15]. Then we get the derived equivalence between dg algebras $\operatorname{Hom}_{A}^{\bullet}\left(A \oplus P_{X_{r}}^{\bullet}, A \oplus P_{X_{r}}^{\bullet}\right)$ and $\operatorname{Hom}_{A}^{\bullet}\left(A \oplus P_{X_{n-r}}^{\bullet}, A \oplus P_{X_{n-r}}^{\bullet}\right)$, and their cohomology rings $\mathrm{E}^{\mathbb{N}}\left(A \oplus X_{r}\right)$ and $\mathrm{E}^{\mathbb{N}}\left(A \oplus X_{n-r}\right)$ are also derived equivalent.

Acknowledgements. S. Y. Pan is supported by National Natural Science Foundation of China (No. 11201022), the Fundamental Research Funds for the Central Universities (2015JBM101) and the 111 Project of China (No. B16002). The authors thank Prof. Wei Hu for his useful suggestions and discussions.

\section{References}

[Bri99] T. Bridgeland, Equivalences of derived categories and fourier-mukai transforms, Bull. Lond. Math. Soc. 31 (1999), 25-3.

[CY15] X. ChEn and D. YANG, Homotopy categories, Leavitt path algebras and Gorenstein projective modules, Intrer. Math. Res. Notice 2015 (2015), 2597-2633.

[Dug15] A. Dugas, A construction of derived equivalent pairs of symmetric algebras, Proc. Amer. Math. Soc. 143 (2015), 2281-2300.

[ELO09] A. I. Efimov, V. A. LunTs, and D. O. Orlov, Deformation theory of objects in homotopy and derived categories I: General theory, Adv. Math. 222 (2009), 359-401.

[Hap88] D. HAPPEL, Triangulated categories in the representation theory of finite dimensional algebras, 119, Cambridge University Press, 1988.

[HK03] M. Hoshino and Y. Kato, Tilting complexes associated with a sequence of idempotents, J. Pure Appl. Algebra 183 (2003), 105-124.

[HP17] W. Hu and S. Y. PAN, Stable functors of derived equivalences and gorenstein projective modules, Math. Nach. 290 (2017), 1512-1530.

[HX10] W. Hu and C. C. XI, Derived equivalences and stable equivalences of Morita type, I, Nagoya Math. J. 200 (2010), 107-152.

[HX13] W. Hu and C. C. XI, Derived equivalences for $\Phi$-Auslander-Yoneda algebras, Trans. Amer. Math. Soc. 365 (2013), 5681-5711.

[IT13] O. Iyama and R. TaKahashi, Tilting and cluster tilting for quotient singularities, Math. Ann. 356 (2013), 10651105.

[Ke194] B. Keller, Deriving dg categories, Ann. Sci. École Norm. Sup. 27 (1994), 63-102. 
[Kel06] B. Keller, On differential graded categories, in International Congress of Mathematicians. Vol. II, Eur. Math. Soc., Zürich, 2006, pp. 151-190.

[Nee01] A. Neeman, Triangulated categories, no. 148, Princeton University Press, 2001.

[NS12] P. Nicolas and M. SAorin, Generalized tilting theory, Appl. Categor. Struct. 26 (2018), 309-368.

[NS14] P. Nicolas and M. Saorin, Classical derived functors as fully faithful embeddings, arXiv preprint arXiv:1403.4726 (2014).

[Pan14] S. Y. PAN, Derived equivalences for $\Phi$-Cohen-Macaulay Auslander-Yoneda algebras, Algebr. Represent. Theory 17 (2014), 885-903.

[PSY14] M. Porta, L. Shaul, and A. Yekutieli, Completion by derived double centralizer, Algebr. Represent. Theor. 17 (2014), 481-494.

[Ric89] J. RickARD, Morita theory for derived categories, J. London Math. Soc. 39 (1989), 436-456.

[Ric91] J. RickARD, Derived equivalences as derived functors, J. London Math. Soc. 43 (1991), 37-48.

[Zhe15] R. ZHENG, On the Auslander-Yoneda algebras of modules over $k[x] /\left(x^{n}\right)$, Algebra Collq. 22 (2015), $147-162$.

Shengyong Pan

Department of Mathematics, Beijing Jiaotong University, 100044 Beijing, China

Beijing Center for Mathematics and Information Interdisciplinary Sciences, 100048 Beijing, China

Email: shypan@jtu.edu.cn

Zhen Peng

School of Mathematics and Statistics, Anyang Normal University, 455000 Anyang, China

Email: pzhen2002@163.com

Jie Zhang

School of Mathematics and Statistics, Beijing Institute of Technology, 100081 Beijing, China

Email: jiezhang@bit.edu.cn 